\title{
Efficient Spectral Domain Analysis of Generalized Multistrip Lines in Stratified Media Including Thin, Anisotropic, and Lossy Substrates
}

\author{
Gabriel Cano, Francisco Medina, Member, IEEE, and Manuel Horno, Member, IEEE
}

\begin{abstract}
This paper deals with the full-wave analysis of multiconductor microstrip lines used in electrooptic modulators (EOM), MMIC or high speed VLSI applications. An arbitrary number of coupled coplanar strips are embedded in a stratified medium involving iso/anisotropic dielectric and/or semiconductor layers. The numerical aspects of the computation of the propagation constants using the spectral domain analysis (SDA) are stressed. An efficient scheme is used to accurately compute attenuation and propagation constants and current distributions with reasonable CPU times. Convergence problems due to the existence of very thin layers adjacent to the metallized interface has been explicitly considered. An algorithm to compute the modal characteristic impedances is provided regardless of the number and nature of substrate layers. A reciprocity related definition of modal impedances is used in this paper. The use of this definition ensures the symmetry of the multiport scattering matrix associated to the structure.
\end{abstract}

\section{INTRODUCTION}

M ICROSTRIP-like transmission lines are the most widely used in MIC and MMIC circuits. They also find applications in electrooptic modulators, SAW transducers and high-speed VLSI interconnects. Consequently, a large amount of technical literature has been devoted to the analysis of these transmission lines. First of all, lossless isotropic dielectric substrates were assumed by most of authors. This is a good assumption for conventional MIC substrates. However, technological advances make it necessary to study the propagation of the electromagnetic field in those transmission lines when printed on a variety of anisotropic dielectric or semiconductor substrates. Dielectric anisotropy often has to be considered (e.g., silicon-on-sapphire technology, electrooptic modulators and certain MIC substrates). A detailed review of the role of anisotropy in integrated circuit structures was reported in [1], and more recent works on this subject can be found in [2]-[5] and references therein. In addition, semiconductor materials with a wide range of doping levels are used in monolithic technology: slowwave transmission lines (Schottky contact or MIS-metalinsulator-semiconductor-structures) are good examples

Manuscript received April 25, 1991; revised September 18, 1991. This work was supported by CICYT Project No. PB87-0798, Spain.

The authors are with the Department of Electronics and Electromagnetics, University of Seville, Avenida Reina Mercedes, s/n, 41012 Seville, Spain.

IEEE Log Number 9104777 of its utilization. In general, MMIC and VLSI technologies imply the use of semiconducting materials submitted to selective doping process. The geometry of these configurations consists of conducting strips printed on lossy stratified substrates. Different methods have been developed to analyze single and coupled planar structures including lossy media. Perturbational methods are precluded because the small losses condition is not fulfilled by semiconductor materials. The simple and analytical parallel-plate waveguide model was initially used for the study of slow-wave structures [6]-[8]. A quasi-TEM approach based on physical considerations has been used in [5], [9], [10]. However, for arbitrary geometries, if high conductivity materials or high frequencies are involved, more accurate theoretical models become necessary. Thus, full-wave approaches were developed later, e.g. mode matching [11], space-domain analysis [12], method of lines [13] and spectral-domain analysis (SDA) [10], [11], [14], [15], [16], [17]. The SDA is probably the most simple and accurate procedure to deal with planar lines. However, from a numerical standpoint, the application of the SDA to the analysis of microstrip lines used in MMIC, high-speed VLSI applications and EOM shows certain important troubles. Part of this paper concerns this subject.

The general multistrip structure studied in this paper is shown in Fig. 1. In the most general case, the substrates are characterized by the following diagonal complex permittivity tensor $\left[\epsilon_{e q}\right]$ :

$$
\left[\epsilon_{e q}\right]=\epsilon_{0}\left(\epsilon_{x} \hat{x} \hat{x}+\epsilon_{y} \hat{y} \hat{y}+\epsilon_{z} \hat{z} \hat{z}\right) ;
$$

with

$$
\epsilon_{\alpha}=\epsilon_{\alpha \alpha_{r}}-j \frac{\sigma_{\alpha \alpha}}{\omega \epsilon_{0}}
$$

where $\alpha$ stands for $x, y, z$, which allows the consideration of anisotropic permittivity (dielectrics) and/or conductivity (semiconductors, semiinsulators). Perfect zero thickness conductors are also assumed. As it was stated above, our goal is to implement an efficient SDA for this problem. When Galerkin's method is used, the most time consuming part of the analysis is the generation of the Galerkin matrix. This matrix must be computed several times before an eigenvalue is attained. This process can be 


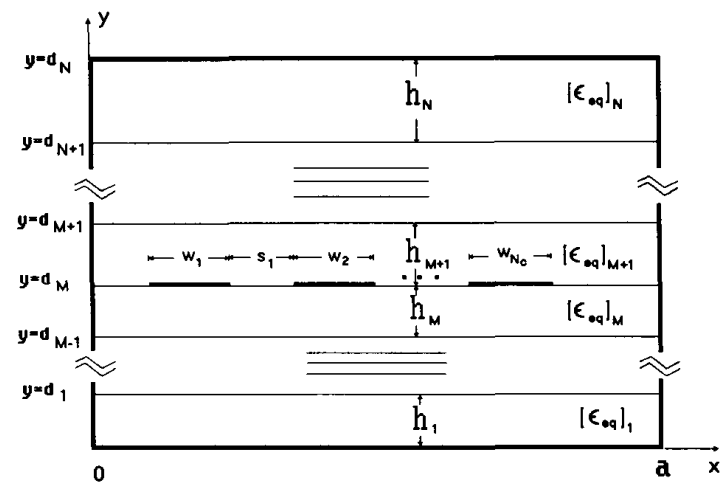

Fig. 1. Transverse section of a multilayered, multiconductor coplanar transmission line with anisotropic dielectrics and semiconductors $\left(\left[\epsilon_{c u}\right]\right)$.

drastically accelerated if an appropriate approximation of the dyadic Green's function is used. Thus, as it will be shown later, the CPU time is kept within reasonable margins and the accuracy of the results is ensured. This method is particularly advantageous when extremely thin layers are adjacent to the metallized interface, since this configuration causes poor convergence when usual techniques are used. The practical significance of this case (e.g., MIS lines, EOM with silica buffer) justifies the analytical effort to overcome this difficulty.

In addition to the computation of the propagation constants, a general procedure to compute the modal impedances is provided. As it is well known, the existence of longitudinal field components supported by non-TEM structures does not allow unique current $I$ and voltage $V$ definitions in terms of path integrals of the fields. The impedance definition for this kind of transmission lines is affected by the aforementioned ambiguity, and the usual TEM definitions are no longer equivalent, showing different dispersive behavior [18]. The adequacy of the definition of the characteristic impedance for microstrip-like or other non-TEM structures has been the subject of discussion by several authors [19]-[21]. In any case, the power flowing through the cross section of the structure is essential to define impedance parameters in single or multiconductor cases, as he has been discussed by different authors [13], [16], [22]-[24]. To our knowledge, the power flux has not been computed as directly as the propagation constant $(\beta)$ in a multilayered system. Just a few examples of explicit treatment of the power flux are found in the literature. As an example, Chen et al. [13] use the method of lines to analyze microstrip lines providing expressions to compute transmitted and loss powers. A new general method to obtain the power flowing through the cross-section of an arbitrary layered structure is shown here. The power flux is explicitly expressed in terms of the surface current distribution on the strips for each propagating mode. The computation of the modal power in a multiconductor line in conjunction with the current eigenvectors make it possible to obtain the modal impedances by using the definition reported in [23] and [24]. This definition is compatible with fundamental reciprocity re- quirements, and differs at high frequencies from other commonly used alternatives (see, for instance, [22]).

Finally, the validity of the numerical results has been checked with previous theoretical and experimental data. From these results it can be concluded that the SDA is a numerically efficient and accurate technique to deal with these sorts of transmission lines if some precautions are taken. In particular, the feasibility of this method to deal with high conductivity substrates is proven. An exhaustive study about the number of trial functions to be retained has been achieved. Although propagation constants are not too sensitive to the number of trial functions in most cases, a good knowledge of the current distribution on the strips is crucial to attain accurate values of modal impedances.

\section{Statement of the Problem: Computation of the Complex Propagation Constants}

The cross section of the multiconductor transmission system considered in this paper is shown in Fig. 1. A wide variety of MMIC (and MIC) transmission lines are particular cases of this general configuration. Since the structure is homogeneous in the $z$-direction, the phasor associated to the electromagnetic field has the following form: $\boldsymbol{A}=\boldsymbol{A}(x, y) e^{-j \gamma z}$. The propagation constant is a complex number, $\gamma(\omega)=\beta(\omega)-j \alpha(\omega), \beta$ being the phase constant, $\alpha$ the attenuation factor, and $\omega$ the angular frequency.

\section{A. Spectral Domain Formulation}

The analytical procedure to determine $\gamma(\omega)$ is the same that the one reported in [4]. There, lossless structures were treated. The extension to the lossy case is carried out here by introducing in the formulation in [4], the complex permittivity tensor (1), and the complex propagation constant, $\gamma$. The method is a SDA where the spectral dyadic Green's function, $\overline{\boldsymbol{G}}(n, \omega)$, is determined via the transverse propagation matrix technique (TPM) [4]. The only difference is the complex nature of all quantities. Permittivities appearing in those expressions become complex quantities, taking into account the lossy nature of media, as it is shown in (1). Fields and currents are then connected in the spectral domain as follows:

$$
\tilde{E}_{M}(n)=j \omega \mu_{0} \bar{G}(n, \omega) \cdot \tilde{J}_{M}(n)
$$

where subindex $M$ refers to the $M$ th interface where conducting strips are printed.

Once $\bar{G}(n, \omega)$ is obtained, Galerkin's method is used to solve the integral equation in the spectral domain. This method leads to a homogeneous system of linear equations with a certain coefficient matrix $[A(\omega, \gamma)]$. The entries of $[A(\omega, \gamma)]$ are numerical series whose general term is

$$
\begin{array}{r}
A_{j k}=\sum_{n} \tilde{J}_{\alpha l}(n) \tilde{J}_{\beta_{m}}^{*}(n) G_{\alpha \beta}(n) ; \\
(\text { where } \alpha, \beta \equiv x, z)
\end{array}
$$

where $\tilde{J}_{\alpha, l}(n)$ is the Fourier transform of the corresponding basis function used to expand the unknown surface 
current density. The set of trial functions used in this work is the same that the one used in [4]. These functions include the strip edge condition and make use of the quasiminimax property of Chebyshev polynomials. The Fourier transforms of these basis functions are known in closed form in terms of the Bessel functions of first kind, which are suitable for numerical treatment.

The condition of nontrivial solution for the equations system, det $[A(\omega, \gamma)]=0$ is, implicitly, the dispersion equation $\gamma=\gamma(\omega)$ of the structure. Its solution provides the mode propagation constants of the structure. Since (3) is a very slowly convergent serie, the solution of the eigenvalue equation is a very slow process. In order to accelerate this part of the analysis some points should be taken into account, as it will be shown in the next subsection.

\section{B. Numerical Solution of the Eigenvalue Equation}

The numerical treatment of the problem is substantially different from that used in [4]. In the general case, solving the eigenvalue equation implies to find the zeroes of a complex function of complex variable. This is achieved by using an effective numerical method which requires to calculate repeatedly the $[A(\omega, \gamma)]$ matrix. These computations should be efficiently achieved to get low CPU times and high accuracy. In this way, it has been found necessary to use a technique to accelerate the convergence of the series involved in (3). The technique is essentially based on the addition and subtraction of approximate series that asymptotically match the series to be added. The main advantage lies in the fact that the approximate series need to be computed only once. Thus, the elements of the Galerkin's matrix are computed as follows:

$$
A_{j k}=\sum_{n} \tilde{J}_{\alpha_{i}}(n) \tilde{J}_{\beta_{m}}^{*}(n)\left\{G_{\alpha \beta}(n)-G_{\alpha \beta}^{\infty}(n)\right\}+K_{j k}
$$

where $K_{j k}$ is the summation of the approximate series.

$$
K_{j k}=\sum_{n} \tilde{J}_{\alpha \prime}(n) \tilde{J}_{\beta_{m}}^{*}(n) G_{\alpha \beta}^{\infty}(n)
$$

and $G_{\alpha \beta}^{\infty}$ is an approximation of the corresponding Green's function element. This approximation is better for increasing values of $n$ (in fact, $\overline{\boldsymbol{G}}(n \gg N) \rightarrow \overline{\boldsymbol{G}}^{\infty}(n)$ ). The complete expression for the elements of $\bar{G}^{\infty}$ is

$$
\begin{aligned}
G_{x x}^{\infty}(n)= & \frac{\alpha_{n}}{k_{0}^{2} \epsilon(n)}\left\{1-\frac{1}{2 \alpha_{n}^{2}}\left[\frac{\gamma^{2} \eta(n)}{\epsilon(n)}+\frac{k_{0}^{2} \xi(n)}{\epsilon(n)}\right]\right\} \\
G_{x z}^{\infty}(n)= & \frac{\gamma}{k_{0}^{2} \epsilon(n)}\left\{1-\frac{1}{2 \alpha_{n}^{2}}\left[\frac{\gamma^{2} \eta(n)}{\epsilon(n)}\right.\right. \\
& \left.\left.+k_{0}^{2}\left(\frac{\xi(n)}{\epsilon(n)}-\frac{2 \psi(n)}{\Phi(n)}\right)\right]\right\} \\
G_{z z}^{\infty}(n)= & \frac{1}{\alpha_{n}}\left\{\left(\frac{\gamma^{2}}{k_{0}^{2} \epsilon(n)}-\frac{1}{\Phi(n)}\right)-\frac{1}{2 \alpha_{n}^{2}}\left[\frac{\gamma^{4} \eta(n)}{k_{0}^{2} \epsilon^{2}(n)}\right.\right. \\
& +\gamma^{2}\left(\frac{1}{\Phi(n)}+\frac{\xi}{\epsilon^{2}(n)}-\frac{4 \psi(n)}{\epsilon(n) \Phi(n)}\right) \\
& \left.\left.+\frac{k_{0}^{2} \vartheta(n)}{\Phi^{2}(n)}\right]\right\}
\end{aligned}
$$

where $\alpha_{n}=n \pi / a$ is the Fourier variable; $k_{0}^{2}=\omega^{2} \mu_{0} \epsilon_{0}$. The functions $\epsilon(n), \eta(n), \vartheta(n), \xi(n), \psi(n)$, and $\Phi(n)$ are computed via readily programmable recurrence expressions (parallel to the ones used in the computation of the Green's dyad) in terms of $n$ and the permittivity tensors of the substrates (see Appendix I). The unknown propagation constant, $\gamma$, appears as a multiplier factor in these expressions. Thus, when $\gamma$ changes in the zero searching process, the approximate series do not have to be computed again. The formula (6) is a cumbersome expression because it is absolutely general and takes into account the presence of an arbitrary number of thin or thick layers. It could be significantly simplified for particular structures with two or three layers or without thin layers. Nevertheless, we have used the complete expression to write a computer code which is useful for the general configuration studied in this paper.

The approximate dyad $\bar{G}^{\infty}(n)$ has been obtained from the truncation of the power series expansion of the eigenvalues $\gamma_{c_{i}}(n), \gamma_{\pi_{i}}(n)$, associated to the $\left[\boldsymbol{K}_{i}(n)\right]$ matrices, and other transversal propagation constants $\gamma_{y_{i}}(n)$ defined in [4]. Terms leading to series in (5) decaying as $1 / n^{5}$ or more quickly have been neglected. In addition, we have eliminated the terms depending on the unknown propagation constant, $\gamma$, in the arguments of the hyperbolic functions involved in the computation of $\overline{\boldsymbol{G}}(n, \omega)$. We must recognize here that a somewhat similar scheme has been used in [25]. However, the study in that paper is limited to a two-layer lossless problem and neglects terms from $1 / n^{3}$. The consequences of this will be discussed in the results section.

It should be noted that the approximate Green's function converges to its true value independently of the ratio between the layer thickness and the box width. Consequently, the first term in (4) becomes a very quickly convergent series which can be added with extremely high accuracy by retaining at most a few tens of Fourier terms (for non critical cases less than ten terms are enough). The accuracy is significantly improved here in comparison with other techniques since the residual error of tails corresponds to a Fourier series decaying as $1 / n^{5}$. In particular, convergence problems in SDA computations owing to the existence of very thin layers are avoided. An arbitrary number of thin substrates or superstrates can be accommodated. The summation in (5), requires to add up much more terms, although asymptotic techniques have been used to accelerate this summation. But, since the dependence on $\gamma$ and $n$ of $G_{\alpha \beta}^{\infty}$ is separated, these series are computed only once for each geometry and each frequency value.

\section{Power Flow and Modal Impedances}

\section{A. Computation of the Complex Power Flux}

The existence of $N_{c}$ conducting strips printed at the $M$ th interface, implies that $N_{c}$ quasi-TEM type fundamental modes can be supported by the structure. Once the modal complex propagation constants $\gamma_{1}, \cdots, \gamma_{N_{c}}$ are computed, a complex modal power associated to each propa- 
gating mode is defined as the flux of the Poynting's vector through the cross-section of the structure:

$$
P^{k}=\frac{1}{2} \iint_{S}\left(\boldsymbol{E} \times \boldsymbol{H}^{*}\right)_{k} \cdot d \boldsymbol{S} .
$$

It should be noted that real and imaginary parts are retained in this definition, in accordance with the theory described in [26] and [24].

From Parseval's theorem, the power flowing through the $i$ th layer is expressed in terms of the transformed electric and magnetic fields in this layer:

$P_{i}^{k}=\frac{a}{2} \sum_{n=-\infty}^{\infty} \int_{d_{i-1}}^{d_{i}}\left[\tilde{E}_{i}(n, y) \times \tilde{\boldsymbol{H}}_{i}^{*}(n, y)\right]_{k} \cdot z_{3} d y$.

Now introducing the expressions in [4] for the electromagnetic fields and the matrix formulation provided by the SDA-TPM scheme, the integration of the Poynting vector over the $y$-variable is analytically carried out by using well known matrix algebra theorems. This mathematical procedures leads to an expression for $P_{i}^{k}$ in function of the transformed tangential electrical fields at the surfaces limiting the $i$ th layer, $\tilde{E}_{i}(n)$ and $\tilde{E}_{i-1}(n)$ :

$$
\begin{aligned}
P_{i}^{k}= & \frac{a}{2 \omega \mu_{0}} \sum_{n=-\infty}^{\infty}\left[\begin{array}{ll}
\tilde{\boldsymbol{E}}_{i}^{T} & \tilde{\boldsymbol{E}}_{i-1}^{T}
\end{array}\right] \\
& \cdot\left[\boldsymbol{M}_{i}\left(n, \omega, \gamma_{k}\right)\right] \cdot\left[\begin{array}{l}
\tilde{\boldsymbol{E}}_{i}^{*} \\
\tilde{\boldsymbol{E}}_{i-1}^{*}
\end{array}\right] .
\end{aligned}
$$

Superscripts $T$ and $*$ standing for transpose and complex conjugate. $\left[\boldsymbol{M}_{i}\left(n, \omega, \gamma_{k}\right)\right]$ is a $4 \times 4$ matrix, which is formed by two $2 \times 2$ matrices arranged in the following way:

$$
\left[M_{i}\left(n, \omega, \gamma_{k}\right)\right]=\left[\begin{array}{rr}
{\left[M_{i}^{A}\right]} & -\left[M_{i}^{B}\right] \\
-\left[M_{i}^{B}\right] & {\left[M_{i}^{A}\right]}
\end{array}\right]
$$

Formally, the $\left[\boldsymbol{M}_{i}^{A}\right]$ and $\left[\boldsymbol{M}_{i}^{B}\right]$ matrices are hyperbolic cotangent and cosecant functions, as is shown in the Appendix II.

The tangential field at $i$ th interface is related to the electric field at the conductor interface via a transfer matrix, $[T(n)]_{j}$, which is readily computed from the $\left[L^{B, U}(n)\right]_{m}$ and $[\boldsymbol{g}(n)]_{m, m \pm 1}$ matrices defined in [4] (see Appendix III). Thus, the power flowing through an arbitrary layer is expressed as a function of the tangential electric field at the $M$ th interface. Introducing (2), we define for each layer a $2 \times 2$ matrix, $\left[N_{i}(n)\right]$ :

$$
\begin{aligned}
{\left[N_{i}\left(n, \omega, \gamma_{k}\right)\right]=} & \bar{G}^{T} \cdot\left[[T]_{i}^{T}[T]_{i-1}^{T}\right] \\
& \cdot\left[M_{i}\right] \cdot\left[\begin{array}{l}
{[T]_{i}^{*}} \\
{[T]_{i-1}^{*}}
\end{array}\right] \cdot \bar{G}^{*} .
\end{aligned}
$$

Thus, the modal power flowing through the $i$ th layer is given by

$$
P_{i}^{k}=\frac{a \omega \mu_{0}}{2} \sum_{n=-\infty}^{\infty} \tilde{J}_{M}^{T} \cdot\left[N_{i}\left(n, \omega, \gamma_{k}\right)\right] \cdot \tilde{J}_{M}^{*}
$$

Finally, the total complex power associated to the $k$ th propagation mode flowing through the cross section of the whole structure, can be expressed in terms of the Fourier transform of the surface current distribution on the conductors:

$$
P^{k}=\frac{a \omega \mu_{0}}{2} \sum_{n=-\infty}^{\infty} \tilde{J}_{M}^{T} \cdot\left[N\left(n, \omega, \gamma_{k}\right)\right] \cdot \tilde{J}_{M}^{*}
$$

where $[N]$ is obtained by adding the $\left[N_{i}\right]$ matrices corresponding to each layer. Note that $\tilde{J}_{M}$ is a known quantity once the eigenvalue problem has been solved and the complex vector solution to the Galerkin's problem has been found. It should be emphasized that $[N(n, \omega, \gamma)]$ is generated by means of a recurrent algorithm working parallel to that used in the Green's dyad computation. Simple $2 \times 2$ matrices operations are involved. Thus, the above expressions turns out to be easily implemented as a computer subroutine allowing the computation of the complex modal power in very general cases of coplanar lines on multilayered substrates.

\section{B. Characteristic and Modal Impedances}

Once the modal power flux is computed, the impedance definition problem is posed. Brews [26] shows the feasibility of imposing the usual current-voltage $(I-V)$ relation for the complex power-even for a hybrid modefor a single microstrip line. If this requirement is fulfilled, the three common definitions-in terms of power-current $(P-I)$, voltage-power $(V-P)$ and voltage-current $(V-I)$ ratio-become equivalent. The uncertainty does not stem from the choice of definition, but from the ability to define one of the quantities, $I$ or $V$. The accessibility of either $I$ or $V$ determines the choice of one of them as the prevailing one.

Different approaches have been used to define the modal impedances of multiconductor lines. For example, definitions chosen in [16] and [22] assign partial powers to the lines. The line-mode impedances are defined as ratios between power and current associated to each line for each mode. Kitazawa in [3] uses a voltage-current definition, since he considers that the total power flux cannot be allocated to the individual strips. However, this definition is arbitrary in some extent [26]. Finally, Wiemer et al. propose in [23] a new definition of modal impedances for multiconductor transmission lines on lossless substrates. A similar requirement to the one used by Brews [26] for the single conductor case is done there. Modal complex powers and voltage and current eigenvectors fulfill the following relationship:

$$
[\boldsymbol{P}]=\frac{1}{2}[\boldsymbol{V}]^{T} \cdot[\boldsymbol{I}]^{*}
$$

where $[\boldsymbol{P}]$ is a diagonal matrix built with the modal powers. Each column of $[V]$ and $[I]$ are the voltage and current eigenvectors associated to each propagation mode. In our case (multistrip line), the primary quantities to be computed are the modal powers and the current eigenvectors $([P]$ and $[I]$ matrices). These are built by taking the 
complex coefficients corresponding to the first term of the current expansion on each conductor, since just this term contributes to the net current with our choice of basis functions. The eigenvoltage matrix $[V]$ is obtained by requiring (14). Modal orthogonality is included in (14) in the lossless case, since $\boldsymbol{V}_{l}^{T} \cdot \boldsymbol{I}_{m}^{*}=\boldsymbol{V}_{l}^{T} \cdot \boldsymbol{I}_{m}=0, l \neq m$. A proof of the suitability of these assumptions can be found in [24], where for a two conductors structure a coupled transmission line model leads to expressions for the modal impedances analogous to the ones provided by the definition given in [23].

Nevertheless, if lossy substrates are present, the above formulation can be used only for certain symmetrical distribution of conductors. In the most general case, the product of the eigenvoltage and the conjugate complex eigencurrent associated to different modes is not zero any more, and the matrix $[\boldsymbol{P}]$ in (14) is non-diagonal. Crosspowers $P^{l m}$ (defined from the electric field of mode $l$ and the magnetic field of mode $m, l \neq m$ ), should be then computed in order to obtain the voltage eigenvectors. Fortunately, the computation of $P^{l, m}, l \neq m$, can be avoided if the orthogonality condition for the eigenvectors is used. So, for a generalized multiconductor microstrip structure, once the eigencurrents and modal powers are obtained for all the independent quasi-TEM type modes, the elements of the voltage eigenvectors are computed by solving the following set of linear equations:

$$
\left.\begin{array}{rl}
P^{k} & =\frac{1}{2} \boldsymbol{V}_{k}^{T} \cdot \boldsymbol{I}_{k}^{*} \\
0 & =\boldsymbol{V}_{l}^{T} \cdot \boldsymbol{I}_{m} ; \quad l \neq \mathrm{m}
\end{array}\right\} \quad k, l, m=1, \cdots, N_{c} .
$$

In this way, only the diagonal elements of $[P]$ have to be computed. This leads to considerable CPU time savings.

Modal impedances are defined for each line, $l$ th, and each mode, $k$ th, as the ratio between the elements of the [V] and [I] matrices, that is $Z_{l k}=V_{l k} / I_{l k}$.

When modal propagation constants, normal mode impedances and current eigenvectors are known, the multiport scattering and impedance matrices of the coupled lines system are easily computed by using the expressions in [22], [27]. The definition of modal impedances contained in this paper preserves the symmetry properties of the scattering and impedance matrices, which stands for a lossy reciprocal system.

\section{Results ANd Discussion}

Before generating reliable numerical results, we have tested the advantages provided by the method proposed in this paper. Firstly, we must emphasize that straightforward addition of the series appearing in the analysis is impractical because thousands of terms should be typically retained, especially if very thin layers are involved. This fact yields prohibitive CPU times in the computation of the complex modal propagation constants. Moreover, the accuracy of the numerical results is strongly dependent on geometrical and electrical parameters. On the contrary, the numerical scheme proposed in (4) in conjunction with (6) makes it feasible to handle configurations having one or several very thin layers in a very efficient manner. Railton et al. proposed a method to consider one thin layer in a lossless problem [25]. First of all, we have generalized that method in order to take into account an arbitrary number of thin layers and lossy materials. This first order approximation of the Green's dyade corresponds to keeping only the first term in (6). However, in many practical cases, several hundreds of Fourier terms must be still retained if only this simplified approximation is used. If a second order approximation is used (namely, the complete expressions in (6)) only a few tens of Fourier terms need to be retained in the worst cases, even if wide boxes and/or high frequency or $\sigma$ values are involved. The quality of the first and second order approximations is compared in Fig. 2 for a particular practical MIS configuration. In this structure a very thin layer of $\mathrm{SiO}_{2}$ lies on a relatively thick $\mathrm{Si}$ substrate. The relative error for $G_{z z}^{\infty}, \Delta G_{z z}(n)=\mid\left(G_{z z}(n)-\right.$ $\left.G_{z z}^{\infty}(n)\right) / G_{z z}(n) \mid$, is plotted in Fig. 2(a) versus the spectral variable $(n)$ for two values of $\sigma$. In Fig. 2(b) we have done the same for three frequency values. The quality of the approximation gets worse when $\sigma$ increases, but anyway, the second order approximation is significantly superior to the first order one. We can also observe another important fact: the superiority of the second order approximation becomes more evident when frequency (and/or box width) increases. These factors negatively affect the convergence of the first order approximation, while the second order is almost unaffected. In fact, when high frequencies or large box widths $(a)$ are involved, the use of the second order approximation is essential. The same conclusions are valid for $\Delta G_{x z}$ and $\Delta G_{x x}$.

The computer codes implemented on the basis of the theory in this paper have been exhaustively checked by comparing with data available in the literature. These data were obtained by means of quasi-TEM (valid in the low frequency range) or other full-wave methods (WienerHopf's technique, spatial domain techniques and so on). We can conclude from these comparisons that the reliability and accuracy of our results is very satisfactory in all cases. In order to illustrate the applications of the technique proposed in this paper, several structures are analyzed and discussed in the next paragraphs.

In Fig. 3, we show the normalized wavelength, $\lambda / \lambda_{0}$ $\left(=k_{0} / \beta\right),-\lambda_{0}$ is the free space wavelength-, and attenuation factor, $\alpha$, for a MIS configuration consisting of a boxed microstrip on $\mathrm{Si}-\mathrm{SiO}_{2}$ substrate. Three different values of Si conductivity are considered and some experimental data reported in [6] are included. By comparing with the results reported in [Fig. 3 in [12]] for the same structure, we can conclude that the agreement of our results with experimental data is slightly more satisfactory than those provided by spatial-domain or finite-elements methods. The slow-wave region is characterized by a large $\left(\lambda / \lambda_{0} \ll<1\right)$ frequency-independent slow-wave factor and by an attenuation constant which is proportional to 


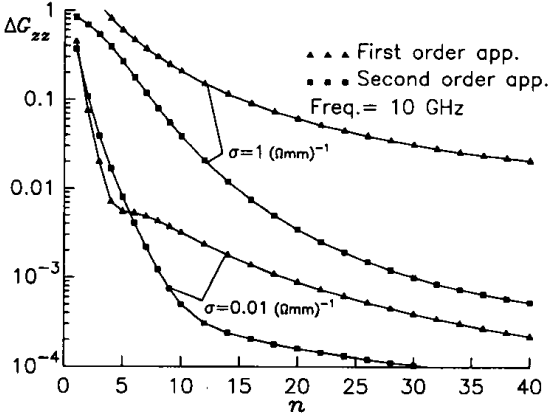

(a)

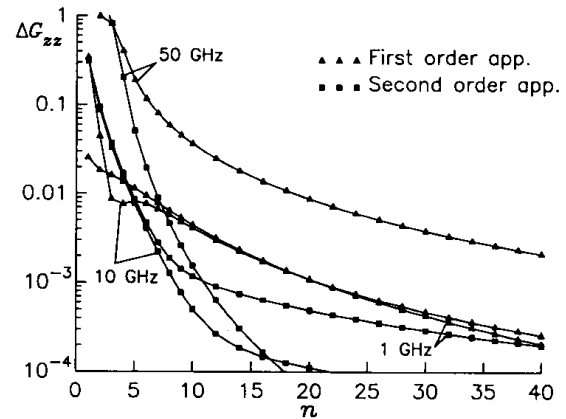

(b)

Fig. 2. Relative error in approximations of $G_{-i}^{\infty}$ for several conductivity and frequency values. The structure is the same that in Fig. 3. (a) Operating frequency: $10 \mathrm{GHz}$. (b) Conductivity: $\sigma_{1}=0.005(\Omega \mathrm{mm})^{-1}$.

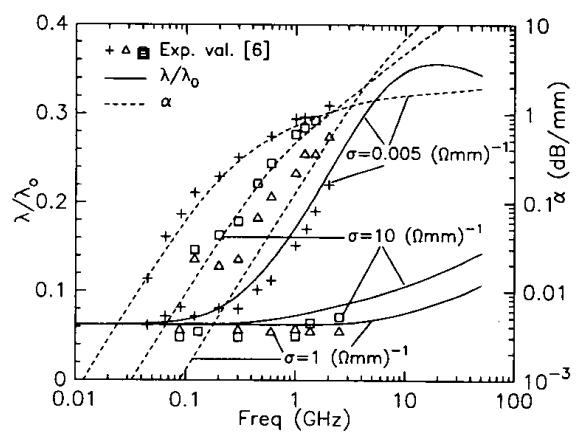

Fig. 3. Dispersion of the normalized wavelength and attenuation factor for a shielded MIS structure for different values of $\sigma_{1}$. Dimensions: $a=10$ $\mathrm{mm} ., h_{1}=0.25 \mathrm{~mm} ., h_{2}=1 \mu \mathrm{m} ., w=160 \mu \mathrm{m} . \epsilon_{r-1}=12, \epsilon_{r-2}=4, \sigma_{2}$ $=0$.

the square of frequency. When frequency increases, the structure enters either in the lossy dielectric region (if $\sigma$ is low enough) or in the skin effect region (if $\sigma$ is large). The characteristic impedance has also been computed by using the three usual definitions (power-current, voltagepower and voltage-current):

$$
Z_{0 I}=2 P /|I|^{2} ; \quad Z_{0 V}=|V|^{2} / 2 P^{*} ; \quad Z_{0 C}=V / I,
$$

being $I$ the longitudinal current flowing on the strip and $V$ the voltage. Here, $V$ is defined as the path integral of the electric field from the center of the strip to the ground plane through a perpendicular straight line. This quantity is computed by using the algorithm proposed in [28] for layered structures, and the power flux by means of the method proposed in this paper. From a computational point of view, it is important to emphasize that more trial functions are necessary in voltage than in power computations, especially in the slow-wave region. This is reasonable from physical intuition. As it was expected, the results obtained show that all the three definitions (both, real and imaginary parts) are indistinguishable at low frequencies (see Fig. 4). However, the frequency behavior differs at higher frequencies. This is because the fundamental relation among power, voltage and current $(P=$ $\left.\frac{1}{2} V \cdot I^{*}\right)$ is not fulfilled by $V$ when defined as the path integral. In accordance with the discussions in Section III-B $V$ should be defined from $I$ and $P$. In the following, we will restrict ourselves to the power/current definition. Experimental data are also plotted in Fig. 4: good agreement is found for these particular geometrical and electrical parameters.

Livernois et al. [12] point out strong qualitative and quantitative discrepancies between their spatial domain results and the SDA results reported in [14]. These discrepancies arise when wide strips and high $\sigma$ are present. They claim that SDA results are wrong because they are too far from the ones computed from a parallel-plate waveguide model. This reasoning is correct since the parallel-plate analysis should work very well for wide strips. However, we have carefully computed the slow-wave factor for this structure and we have found that our results are consistent with the parallel-plate waveguide model (see Fig. 5). In fact, the agreement is better when $\omega$ increases, as it is expected from a physical argument: the smaller the skin depth is, the better the parallel-plate model works. Hence, our results seem to be very reliable. Wrong SDA data stem essentially from two numerical error sources. On the one hand, several basis functions of the kind used in this paper must be retained when wide strips or high $\sigma$ are involved to correctly approximate the solution (the results reported in [14] were computed by using only one function). On the other hand, care must be taken in the evaluation of the series or integrals appearing in the spectral analysis, especially when very thin layers and/or high $\sigma$ materials are involved (as it is the case). In fact, we have also detected significant differences with spectral results in [14] even if only one trial function is used in our computations. This point has been highlighted in this paper. When an adequate number of trial functions and Fourier terms are used the results provided by the method in this paper are closer to the ones computed from the parallel plate model. We have also observed that the spatial domain results reported in [12] for the highest value of $\sigma$ (see Fig. 5) are slightly too high. Similar numerical error sources could explain this disagreement with our results.

Finally we present some results for multistrip struc- 


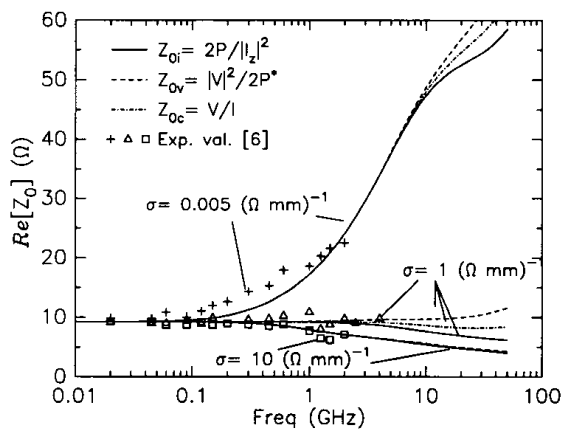

(a)

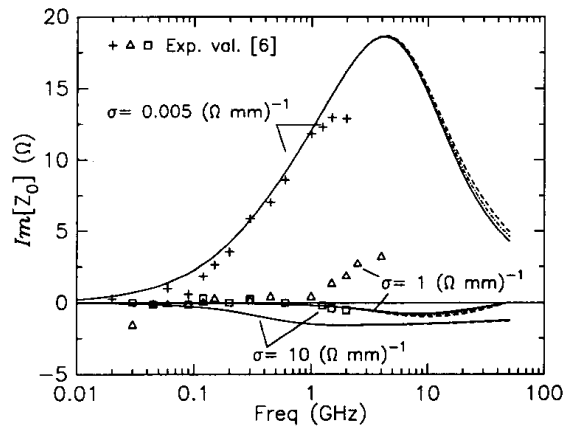

(b)

Fig. 4. Frequency behavior of the (a) real and (b) imaginary parts of the characteristic impedances for the MIS structure in Fig. 3

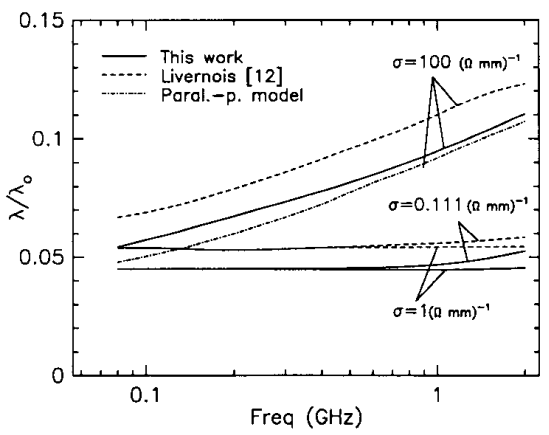

(a)

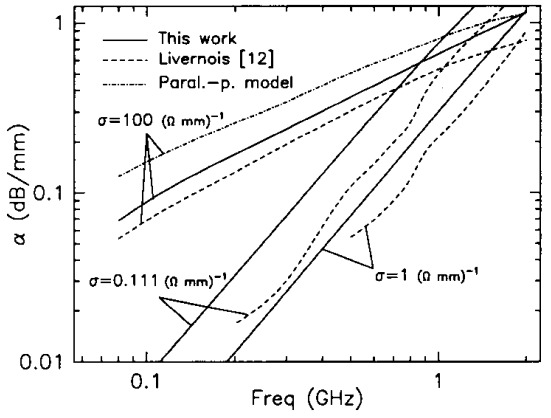

(b)

Fig. 5. Comparison of results for the complex propagation constant obtained by means of three differents methods. Single MIS structure. Dimensions: $a=10 \mathrm{~mm}$., $h_{1}=0.25 \mathrm{~mm} ., h_{2}=1 \mu \mathrm{m} ., w=0.6 \mathrm{~mm} . \epsilon_{r .1}=12$. $\epsilon_{r, 2}=4, \sigma_{2}=0$. (a) Normalized wavelength. (b) Attenuation factor.

tures. Two shielded symmetric coupled microstrips on three layer substrate-insulator-semiconductor-semi-insulator-are first studied. This structure presents two very thin layers located under the conductors. The results for $\beta / k_{0}$ and $\alpha$ compares favorably with data in [16], where a SDA is also used. Modal impedances agree satisfactorily for the less dispersive mode-odd mode-but significant discrepancies are observed at high $\omega$ with even mode data, as it can be seen in Fig. 6. In Fig. 7 we show results for a three conductor structure in a three layers configuration. In Fig. 7(a), slow-wave factors and attenuations are plotted for the three fundamental propagating modes. The plus, minus and zero signs $(+,-, 0)$, stand for the sense of the current flow, $I_{z}$, on the conductors. Mode \#1 presents the lowest $\beta / k_{0}$ and relatively high attenuation in the low-frequency range, owing to the existence of an important electric field in the semiconductor layer. At high frequencies (dielectric modes propagate), it is the field distribution corresponding to mode \# 3 which shows the highest attenuation factor. Real and imaginary parts of the modal impedance $\left[Z_{i j}\right]$ (ith conductor, $j$ th mode), are depicted in 7(b) and (c). It should be noticed that, as in the single microstrip case, the propagation as dielectric mode, $\left(\beta / k_{0} \sim 5\right)$, for each fundamental mode is preceded for

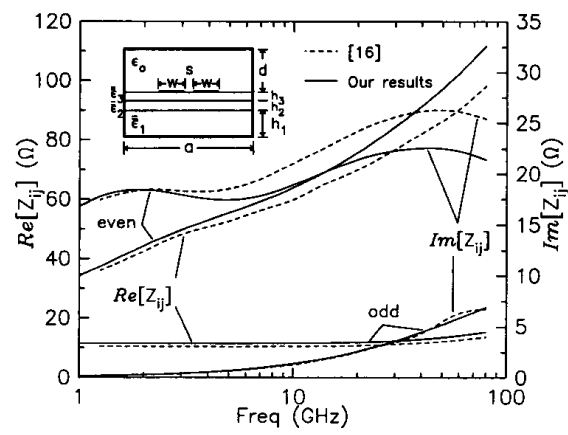

Fig. 6. Real and imaginary parts of modal impedances for two shielded symmetric coupled microstrip on three layer substrate. Dimensions: $a=2$ $\mathrm{mm} ., h_{1}=0.1 \mathrm{~mm} ., h_{2}=0.6 \mu \mathrm{m} ., h_{3}=0.4 \mu \mathrm{m} ., w=10 \mu \mathrm{m} ., s=10$ $\mu \mathrm{m} ., \epsilon_{r, 1}=\epsilon_{r, 2}=\epsilon_{r, 3}=12.7(\mathrm{AsGa}) ; \sigma_{1}=10^{-5}(\Omega \mathrm{mm})^{-1} ; \sigma_{2}=$ $10(\Omega \mathrm{mm})^{-1} ; \sigma_{3}=0$.

a maximum in the imaginary part of the corresponding modal impedances. For design applications, mode current ratios $\left(R_{i j} \equiv I_{i j} / I_{1 j}\right)$, should be provided if a three-conductors transmission line is considered [22]. Obviously, these parameters are known once the current eigenvectors have been computed. 


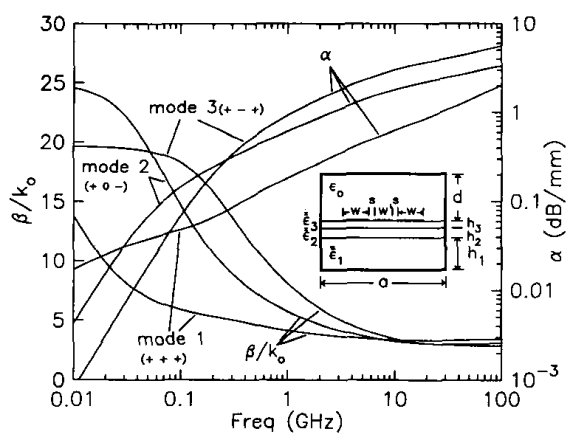

(a)

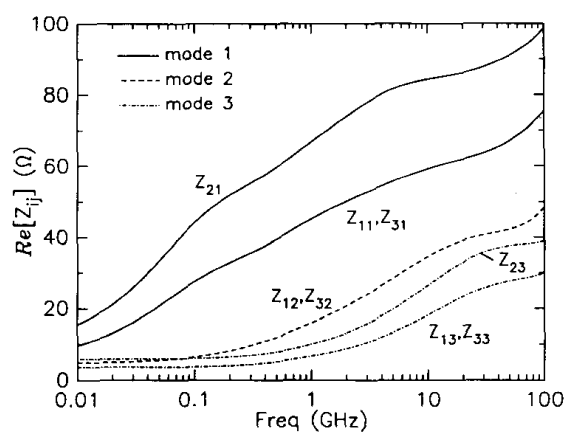

(b)

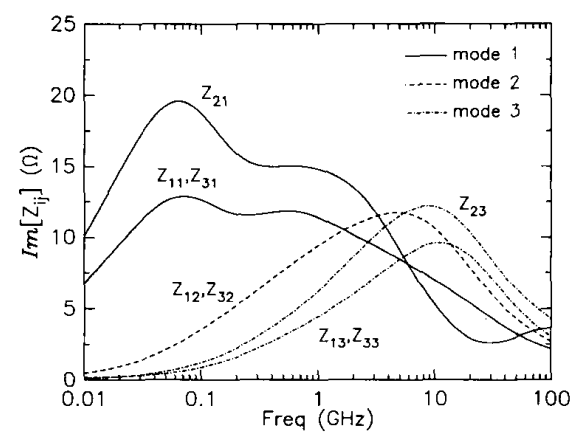

(c)

Fig. 7. Three layers and three conductors configurations. Modal parameters: (a) Slow-wave and attenuation factors. (b) Real parts. (c) Imaginary parts of modal impedances. Dimensions: $a=6 \mathrm{~mm} ., h_{1}=0.2 \mathrm{~mm} ., h_{2}$ $=10 \mu \mathrm{m} ., h_{3}=1 \mu \mathrm{m} ., w=150 \mu \mathrm{m} ., w^{\prime}=100 \mu \mathrm{m} ., s=100 \mu \mathrm{m} . \epsilon_{r 1}=$ $\epsilon_{r 2}=\epsilon_{r 3}=12.7(\mathrm{AsGa}) ; \sigma_{1}=10^{-5}(\Omega \mathrm{mm})^{-1}, \sigma_{2}=5 \cdot 10^{-3}(\Omega-\mathrm{mm})^{-1}$, $\sigma_{3}=0$.

\section{CONCLUSION}

In this paper we have presented a method based on the spectral domain formulation to efficiently analyze single and multiconductor microstrip transmission lines used in MMIC and high-speed VLSI circuits. Certain numerical convergence problems arising in the study of this kind of transmission structures are treated with detail. Approximate expressions for the Green's dyad are used to drastically improve the convergence of the Fourier series involved in the computations. Important CPU time savings are achieved with this procedure. The accuracy and reliability of the results are also meaningfully enhanced. In addition, a general method to compute the power flux through the cross section of the structure has been developed. The power flowing for each propagating mode is expressed as a function of the surface current density on the strips (which is known from the Galerkin's analysis). The expression for the power is valid for an arbitrary number of anisotropic and/or semiconductor layers. Once modal powers and current eigenvectors are computed, voltage vectors are defined from the relation $[P]=\frac{1}{2}[\boldsymbol{V}]^{T}$ - $[I]^{*}$ and the modal orthogonality condition. Modal impedances are obtained now in the usual way as the voltage/current ratio for each line and each mode.

\section{APPENDIX I}

The functions appearing in the definition of the approximate spectral Green's dyad (6), are computed as follows (superscript + stands for $M+1 \leq i<N$, and - for 1 $<i \leq M$, that is, they refer to layers above and below the $M$ th interface respectively):

a) $\Phi(n)=\Phi_{M+1}^{+}(n)+\Phi_{M}^{-}(n)$

$\Phi_{i}^{ \pm}(n)=\frac{1+\operatorname{coth}\left(\alpha_{n} h_{i}\right) \Phi_{i \pm 1}^{ \pm}(n)}{\operatorname{coth}\left(\alpha_{n} h_{i}\right)+\Phi_{i \pm 1}^{ \pm}(n)} ;$

$\Phi_{1}^{-}(n)=\operatorname{coth}\left(\alpha_{n} h_{1}\right) ; \quad \Phi_{N}^{+}(n)=\operatorname{coth}\left(\alpha_{n} h_{N}\right)$.

b) $\epsilon(n)=\epsilon_{M+1} E_{M+1}^{+}(n)+\epsilon_{M} E_{M}^{-}(n)$

$E_{i}^{ \pm}(n)=\frac{\epsilon_{i}+\epsilon_{i \pm 1} E_{i \pm 1}^{ \pm}(n) \operatorname{coth}\left[\alpha_{n} \epsilon_{x y_{i}}^{1 / 2} h_{i}\right]}{\epsilon_{i \pm 1} E_{i \pm 1}^{ \pm}+\epsilon_{i} \operatorname{coth}\left[\alpha_{n} \epsilon_{x y_{i}}^{1 / 2} h_{i}\right]} ;$

$$
\epsilon_{j}=\left(\epsilon_{x_{j}} \epsilon_{y_{j}}\right)^{1 / 2} ; \quad \epsilon_{x y_{j}}=\frac{\epsilon_{x_{j}}}{\epsilon_{y_{j}}}
$$

$E_{1}^{-}(n)=\operatorname{coth}\left[\alpha_{n} \epsilon_{x y_{1}}^{1 / 2} h_{1}\right] ; \quad E_{N}^{+}(n)=\operatorname{coth}\left[\alpha_{n} \epsilon_{x y_{N}}^{1 / 2} h_{N}\right]$.

The general expressions for the functions appearing in 
the second order approximation are

$$
\begin{aligned}
& \text { c) } \xi(n)=\epsilon_{y M+1} \epsilon_{M+1} E_{M+1}^{+}(n)+\epsilon_{y_{M}} \epsilon_{M} E_{M}^{-}(n)-\Xi_{M+1}(n)-\Xi_{M}(n) \\
& \Xi_{M}(n)=\frac{\left(\epsilon_{y M}-\epsilon_{y M-1}\right) \epsilon_{M-1} E_{M-1}^{-}(n) \epsilon_{M}^{2} \operatorname{cosch}^{2}\left[\alpha_{n} \epsilon_{x y M}^{1 / 2} h_{M}\right]}{\left[\epsilon_{M} \operatorname{coth}\left[\alpha_{n} \epsilon_{x y_{M}}^{1 / 2} h_{M}\right]+\epsilon_{M-1} E_{M-1}^{-}(n)\right]^{2}} \quad(M \neq 1) \\
& \Xi_{M+1}(n)=\frac{\left(\epsilon_{y M+1}-\epsilon_{y M+2}\right) \epsilon_{M+2} E_{M+2}^{+}(n) \epsilon_{M+1}^{2} \operatorname{cosch}^{2}\left[\alpha_{n} \epsilon_{x y M+1}^{1 / 2} h_{M+1}\right]}{\left[\epsilon_{M+1} \operatorname{coth}\left[\alpha_{n} \epsilon_{x y M+1}^{1 / 2} h_{M+1}\right]+\epsilon_{M+2} E_{M+2}^{+}(n)\right]^{2}} \quad(M+1 \neq N) . \\
& \text { d) } \eta(n)=\frac{\epsilon_{y M+1} \epsilon_{z M+1} E_{M+1}^{+}(n)}{\epsilon_{M+1}}+\frac{\epsilon_{y M} \epsilon_{z M} E_{M}^{-}(n)}{\epsilon_{M}}-\Delta_{M+1}(n)-\Delta_{M}(n) \\
& \Delta_{M}=\frac{\left(\epsilon_{y_{M}} \epsilon_{z M} \epsilon_{M-1}^{2}-\epsilon_{y_{M-1}} \epsilon_{z M-1} \epsilon_{M}^{2}\right) E_{M-1}^{-} \operatorname{cosch}^{2}\left(\alpha_{n} \epsilon_{x y M}^{1 / 2} h_{M}\right)}{\epsilon_{M-1}\left[\epsilon_{M} \operatorname{coth}\left(\alpha_{n} \epsilon_{x y_{M}}^{1 / 2} h_{M}\right)+\epsilon_{M-1} E_{M-1}^{-}\right]^{2}} \quad(M \neq 1) . \\
& \Delta_{M+1}=\frac{\left(\epsilon_{y M+1} \epsilon_{z M+1} \epsilon_{M+2}^{2}-\epsilon_{y_{M+2}} \epsilon_{z M+2} \epsilon_{M+1}^{2}\right) E_{M+2}^{-} \operatorname{cosch}^{2}\left(\alpha_{n} \epsilon_{x y_{M+1}}^{1 / 2} h_{M+1}\right)}{\epsilon_{M+2}\left[\epsilon_{M+1} \operatorname{coth}\left(\alpha_{n} \epsilon_{x y M+1}^{1 / 2} h_{M+1}\right)+\epsilon_{M+2} E_{M+2}^{+}\right]^{2}} \quad(M+1 \neq N) \\
& \text { e) } \psi(n)=\chi_{M+1}(n)+\chi_{M}(n)-\Psi_{M+1}(n)-\Psi_{M}(n) \\
& \Psi_{M}=\frac{1}{\operatorname{coth}\left(\alpha_{n} h_{M}\right)+\Phi_{M-1}^{-1}(n)}\left\{\Upsilon_{M}^{\prime}(n)\right. \\
& \left.-\frac{\epsilon_{M} \operatorname{cosch}\left(\alpha_{n} h_{M}\right) \operatorname{cosch}\left(\alpha_{n} \epsilon_{x y M}^{1 / 2} h_{M}\right)\left[\Upsilon_{M}^{\prime \prime}(n)+\chi_{M-1}(n)\right]}{\epsilon_{M} \operatorname{coth}\left(\alpha_{n} \epsilon_{x y M}^{1 / 2} h_{M}\right)+\epsilon_{M-1} E_{M-1}^{-}(n)}\right\} \quad(M \neq 1) \\
& \Psi_{M+1}=\frac{1}{\operatorname{coth}\left(\alpha_{n} h_{M+1}\right)+\Phi_{M+2}^{+}(n)}\left\{\Upsilon_{M+1}^{\prime}(n)\right. \\
& \left.-\frac{\epsilon_{M+1} \operatorname{cosch}\left(\alpha_{n} h_{M+1}\right) \operatorname{cosch}\left(\alpha_{n} \epsilon_{x y M+1}^{1 / 2} h_{M+1}\right)\left[\Upsilon_{M+1}^{\prime \prime}(n)+\chi_{M+2}(n)\right]}{\epsilon_{M+1} \operatorname{coth}\left(\alpha_{n} \epsilon_{x y+1}^{1 / 2} h_{M+1}\right)+\epsilon_{M+2} E_{M+2}^{+}(n)}\right\} \quad(M+1 \neq N) \\
& \chi_{i}(n)=\epsilon_{i} E_{i}^{ \pm}(n) \frac{\epsilon_{z_{i}}-\epsilon_{y_{i}}}{\epsilon_{x_{i}}-\epsilon_{y_{i}}}+\epsilon_{y_{i}} F_{i}^{ \pm} \frac{\epsilon_{x_{i}}-\epsilon_{z_{i}}}{\epsilon_{x_{i}}-\epsilon_{y_{i}}} \\
& F_{i}^{ \pm}=\operatorname{coth}\left(\alpha_{n} h_{i}\right)-\frac{\epsilon_{i} \operatorname{cosch}\left(\alpha_{n} h_{i}\right) \operatorname{cosch}\left(\alpha_{n} \epsilon_{x y_{i}}^{1 / 2} h_{i}\right)}{\epsilon_{i} \operatorname{coth}\left(\alpha_{n} \epsilon_{x y_{i}}^{1 / 2} h_{i}\right)+\epsilon_{i \pm 1} E_{i \pm 1}^{ \pm}(n)} \\
& F_{1}^{-}(n)=\operatorname{coth}\left(\alpha_{n} h_{1}\right) ; \quad F_{N}^{+}(n)=\operatorname{coth}\left(\alpha_{n} h_{N}\right) \text {. } \\
& \Upsilon_{i}^{\prime}(n)=\epsilon_{i} \operatorname{cosch}\left(\alpha_{n} h_{i}\right) \operatorname{cosch}\left(\alpha_{n} \epsilon_{x y_{i}}^{1 / 2} h_{i}\right) \frac{\epsilon_{z_{i}}-\epsilon_{y_{i}}}{\epsilon_{x_{i}}-\epsilon_{y_{i}}}+\epsilon_{y_{i}} \operatorname{cosch}^{2}\left(\alpha_{n} h_{i}\right) \frac{\epsilon_{x_{i}}-\epsilon_{z_{i}}}{\epsilon_{x_{i}}-\epsilon_{y_{i}}} \\
& \Upsilon_{i}^{\prime \prime}(n)=\epsilon_{i} \operatorname{coth}\left(\alpha_{n} \epsilon_{x y_{i}}^{1 / 2} h_{i}\right) \frac{\epsilon_{z i}-\epsilon_{y_{i}}}{\epsilon_{x_{i}}-\epsilon_{y_{i}}}+\epsilon_{y_{i}} \operatorname{coth}\left(\alpha_{n} h_{i}\right) \frac{\epsilon_{x_{i}}-\epsilon_{z_{i}}}{\epsilon_{x_{i}}-\epsilon_{y_{i}}} . \\
& \text { f) } \vartheta(n)=\epsilon_{z M+1} \Phi_{M+1}^{+}(n)+\epsilon_{z M} \Phi_{M}^{-}(n)-\theta_{M+1}(n)-\theta_{M}(n) \\
& \theta_{M}(n)=\frac{\left(\epsilon_{z M}-\epsilon_{z M-1}\right) \Phi_{M-1}(n) \operatorname{cosch}^{2}\left(\alpha_{n} h_{M}\right)}{\left[\operatorname{coth}\left(\alpha_{n} h_{M}\right)+\Phi_{M-1}^{-}(n)\right]^{2}} \quad(M \neq 1) \\
& \theta_{M+1}(n)=\frac{\left(\epsilon_{z M+1}-\epsilon_{z M+2}\right) \Phi_{M+2}^{+}(n) \operatorname{cosch}^{2}\left(\alpha_{n} h_{M+1}\right)}{\left[\operatorname{coth}\left(\alpha_{n} h_{M+1}\right)+\Phi_{M+2}^{+}(n)\right]^{2}} \quad(M+1 \neq N)
\end{aligned}
$$

Some of the expressions above are valid for $M \neq 1$ and $M+1 \neq N$. If $M=1$ or $M+1=N$ those expressions are zero.

It must be emphasized that the above expressions in- volve the same recurrence relations used in the computation of $\bar{G}(n, \omega)$. Thus, redundant computations can be avoided. 


\section{APPENDIX II}

In this appendix we define the $\left[\boldsymbol{M}_{i}^{A, B}\right]$ matrices appearing in power computations. Let $\left[K_{i}(n)\right]$ the transverse propagation matrix associated to the $i$ th layer such as defined in [4], and $\left[Q_{i}(n)\right]$ its diagonalization matrix:

$$
\left[\boldsymbol{K}_{i}\right]=\left[\boldsymbol{Q}_{i}\right] \cdot\left[\begin{array}{cc}
\gamma_{c_{i}} & 0 \\
0 & \gamma_{\pi_{i}}
\end{array}\right] \cdot\left[\boldsymbol{Q}_{i}\right]^{-1}
$$

being $\gamma_{c_{i}}(n), \gamma_{\pi_{i}}(n)$ the eigenvalues of $\left[K_{i}(n)\right]$. Then, the $\left[\boldsymbol{M}_{i}^{A}\right],\left[\boldsymbol{M}_{i}^{B}\right]$ matrices in (10) are computed in the following way:

$$
\begin{aligned}
{\left[\boldsymbol{M}_{i}^{A}(n)\right] } & =\left(\left[Q_{i}\right]^{-1}\right)^{t} \cdot\left[A_{i}(n)\right] \cdot\left(\left[Q_{i}\right]^{-1}\right)^{*} \\
{\left[M_{i}^{B}(n)\right] } & =\left(\left[Q_{i}\right]^{-1}\right)^{t} \cdot\left[B_{i}(n)\right] \cdot\left(\left[Q_{i}\right]^{-1}\right)^{*}
\end{aligned}
$$

The elements of $\left[\boldsymbol{A}_{i}\right]$ and $\left[\boldsymbol{B}_{i}\right]$ matrices are

$$
\begin{aligned}
&\left(A_{k l}\right)_{i}= \frac{1}{\gamma_{k_{i}}^{2}-\gamma_{l_{i}}^{* 2}}\left\{\left[\left(C_{k l}\right)_{i}+\left(D_{k l}\right)_{i} \frac{\gamma_{l_{i}}^{* 2}}{\gamma_{y_{i}}^{2}}\right] \gamma_{k_{i}} \operatorname{coth}\left(\gamma_{k_{i}} h_{i}\right)\right. \\
&\left.-\left[\left(C_{k l}\right)_{i}+\left(D_{k l}\right)_{i} \frac{\gamma_{k_{i}}^{2}}{\gamma_{y_{i}}^{2}}\right] \gamma_{l_{i}}^{*} \operatorname{coth}\left(\gamma_{l_{i}}^{*} h_{i}\right)\right\} \\
&\left(B_{k l}\right)_{i}= \frac{1}{\gamma_{k_{i}}^{2}-\gamma_{l i}^{* 2}}\left\{\left[\left(C_{k l}\right)_{i}+\left(D_{k l}\right)_{i} \frac{\gamma_{l_{i}}^{* 2}}{\gamma_{y_{i}}^{2}}\right] \gamma_{k_{i}} \operatorname{cosch}\left(\gamma_{k_{i}} h_{i}\right)\right. \\
&\left.-\left[\left(C_{k l}\right)_{i}+\left(D_{k l}\right)_{i} \frac{\gamma_{k_{i}}^{2}}{\gamma_{y_{i}}^{2}}\right] \gamma_{l_{i}}^{*} \operatorname{cosch}\left(\gamma_{l_{i}}^{*} h_{i}\right)\right\} \\
&(k, l=1,2)
\end{aligned}
$$

where $h_{i}$ is the thickness of the $i$ th layer; $\gamma_{y_{i}}^{2}=\alpha_{n}^{2}+\gamma^{2}$ $-k_{0}^{2} \epsilon_{y_{i}}$, and $\gamma_{1}, \gamma_{2}$ stand for the eigenvalues $\gamma_{c}, \gamma_{\pi}$, respectively. $\left(C_{k l}\right)_{i}$ and $\left(D_{k l}\right)_{i}$ are elements of the following matrices:

$$
\begin{aligned}
& {\left[\boldsymbol{C}_{i}\right]=\left[\boldsymbol{Q}_{i}\right]^{t} \cdot\left[\begin{array}{cc}
\gamma & -\alpha_{n} \\
0 & 0
\end{array}\right] \cdot\left[\boldsymbol{Q}_{i}\right]^{*}} \\
& {\left[\boldsymbol{D}_{i}\right]=\left[\boldsymbol{Q}_{i}\right]^{t} \cdot\left[\begin{array}{cc}
\alpha_{n} & 0 \\
\gamma & 0
\end{array}\right] \cdot\left[\boldsymbol{Y}_{i}\right]^{*} \cdot\left[\boldsymbol{Q}_{i}\right]^{*}}
\end{aligned}
$$

being $\left[Y_{i}\right]$ the admittance matrix defined in [4], which relates the tangential components of the electric and magnetic fields in the spectral domain.

\section{APPENDIX III}

The spectral components of the tangential electric field at the $j$ th interface can be expressed in terms of the electric field at the strips interface $(M \mathrm{th})$ in the following way:

$$
\tilde{E}_{j}=[\boldsymbol{T}(n)]_{j} \cdot \tilde{E}_{M}
$$

where $[\boldsymbol{T}(n)]_{j}$ matrix is given by

$$
[\boldsymbol{T}(n)]_{j}(n)=\left\{\begin{array}{c}
(-1)^{M-j} \prod_{k=j+1}^{M}\left[\boldsymbol{L}^{B}(n)\right]_{k-1}^{-1} \cdot[\boldsymbol{g}(n)]_{k-1, k} \\
\text { if } j<M \\
(-1)^{j-M} \prod_{k=M+1}^{j}\left[\boldsymbol{L}^{U}(n)\right]_{N-k}^{-1} \cdot[\boldsymbol{g}(n)]_{k, k-1} \\
\quad \text { if } j>M
\end{array}\right.
$$

The $\left[\boldsymbol{L}^{B}\right]_{k-1}$ and $\left[\boldsymbol{L}^{U}\right]_{N-k}$ matrices are defined in $[4]$.

\section{REFERENCES}

[1] N. G. Alexopoulos, "Integrated circuit structures on anisotropic substrates," IEEE Trans. Microwave Theory Tech., vol. MTT-33, pp. 847-881, Oct. 1985.

[2] M. Kobayashi and H. Momoi, "Normalized transverse current distributions of microstrip lines on anisotropic substrates." IEEE Trans. Microwave Theory Tech., vol. 36, pp. 1406-1410, Oct. 1988

[3] T. Kitazawa, "Variational method for multiconductor coupled striplines with stratified anisotropic media," IEEE Trans. Microwave Theory Tech., vol. 37, pp. 484-491, Mar. 1989.

[4] F. Medina, M. Horno, and H. Baudrand, "Generalized spectral analysis of planar lines on layered media including uniaxial and biaxial dielectric substrates," IEEE Trans. Microwave Theory Tech., vol. 37, pp. 504-511, Mar. 1989.

[5] M. Horno, F. Mesa, F. Medina, and R. Marqués, "Quasi-TEM anal ysis of multilayered, multiconductor coplanar structures with dielectric and magnetic anisotropy including substrate losses," IEEE Trans. Microwave Theory Tech., vol. 38, pp. 1059-1068, Aug. 1990.

[6] H. Hasegawa, M. Furukawa, and H. Yanai, "Properties of microstrip line on $\mathrm{Si}_{-} \mathrm{SiO}_{2}$ system," IEEE Trans. Microwave Theory Tech., vol. MTT-19, pp. 869-881, 1971.

[7] D. Jäger and W. Rabus, "Bias-dependent phase delay of Schottky contact microstip line," Electron. Lett., vol. 9, no. 10, pp. 201-203, 1973.

[8] K. Sachse and A. Sawicki, "Analytical modelling of MIS and Schottky contact microstrip lines," in Proc. Int. URSI Symp., pt. B, Budapest, 1986, pp. 427-429.

[9] H. Hasegawa and H. Okizaki, "MIS and Schottky slow-wave coplanar striplines on GaAs substrates," Electron. Lett., vol. 13, no. 22, pp. 663-664, 1977.

[10] S. Seki and H. Hasegawa, "Cross-tie slow-wave coplanar waveguide on semi-insulating GaAs substrates," Electron. Lett., vol. 17, no. 25, pp. 940-941, 1981

[11] Y. Fukuoka, Y. C. Shih, and T. Itoh, "Analysis of slow-wave coplanar waveguide for monolithic integrated circuits," IEEE Trans. Microwave Theory Tech., vol. MTT-31, pp. 567-573, July 1983.

[12] T. G. Livernois and P. B. Katehi, "A generalized method for deriving the space-domain Green's function in a shielded, multilayer substrate structure with applications to MIS slow-wave transmission lines," IEEE Trans. Microwave Theory Tech., vol. 37, pp. 17611767, Nov, 1989

[13] Z. Chen and B. Gao, "Full-wave analysis of multiconductor coupled lines in MICs by the method of the lines," IEE Proc. Microwave, Antennas and Propagation, pt. H, vol. 136, no. 5, pp. 399-404, 1989

[14] P. Kennis and L. Faucon, "Rigorous analysis of planar MIS transmission lines," Electron. Lett, vol. 17, no. 13, pp. 454-456, 1981.

[15] Y. C. Shih and T. Itoh, "Analysis of printed transmission lines for monolithic integrated circuits," Electron. Lett., vol. 18, no. 14, pp. $585-586,1982$.

[16] T. C. Mu, H. Ogawa, and T. Itoh, "Characteristics of multiconductor, asymmetric, slow-wave microstrip transmission lines," IEEE Trans. Microwave Theory Tech., vol. MTT-34, pp. 1471-1477, Dec. 1986.

[17] M. I. Aksun and H. Morko, "GaAs on Si a substrate for microwave 
and millimeter-wave monolithic integration," IEEE Trans. Microwave Theory Tech., vol. 36, pp. 160-162, Jan. 1988

[18] J. B. Knorr and A. Tufekcioglu, "Spectral domain calculation of microstrip characteristic impedance," IEEE Trans. Microwave Theory Tech., vol. MTT-23, pp. 725-728, Sept. 1975.

[19] E. F. Kuester, D. C. Chang, and L. Lewin, "Frequency-dependent definitions of microstrip characteristic impedance," in Proc. International URSI Symp., paper 335 B, München 1980

[20] R. H. Jansen and M. Kirschning, "Arguments and an accurate model for the power-current formulation of microstrip characteristic impedance," Arch. Elek. Übertragung, vol. b and 37, Heft 3/4, pp. 108$112,1983$.

[21] W. J. Getsinger, "Measurement and modelling of the apparent characteristic impedance of microstrip," IEEE Trans. Microwave Theory Tech., vol. MTT-31, pp. 624-632, Aug. 1983.

[22] V. K. Tripathi and $H$. Lee, "Spectral domain computation of characteristic impedances and multiport parameters of multiple coupled microstrip lines," IEEE Trans. Microwave Theory Tech., vol. 37, pp. 215-221, Jan. 1989.

[23] L. Wiemer and R. H. Jansen, "Reciprocity related definition of strip characteristic impedance for multiconductor hybrid-mode transmission lines," Microwave and Optical Tech. Letters, vol. 1, no. 1, pp. 22-25, 1988.

[24] N. Faché and D. de Zutter, "New high-frequency circuit model for coupled lossless and lossy waveguide structures," IEEE Trans. Microwave Theory Tech., vol. 38, pp. 252-259, March 1990.

[25] C. J. Railton and J. P. McGeehan, "A rigorous and computationally efficient analysis of microstrip for use as an electro-optic modulator,' IEEE Trans. Microwave Theory Tech., vol. 37, pp. 1099-1104, July 1989.

[26] J. R. Brews, "Characteristic impedances of microstrip lines," IEEE Trans. Microwave Theory Tech., vol. MTT-35, pp. 30-34, Jan. 1987.

[27] R. R. Boix, "Two- and three-dimensional electrostatic study of plana structures in multilayered anisotropic media: Application to the design of MIC," Ph.D. dissertation, University of Seville, Seville, Spain, 1990

[28] G. Cano, F. Medina, and M. Horno, "Frequency dependent characteristic impedance of microstrip and finline on layered biaxial substrates," Microwave and Optical Tech. Letters, vol. 2, no. 6, pp. 210-214, 1989

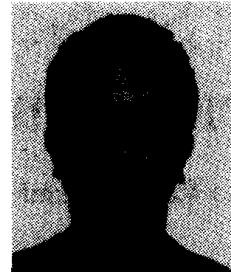

Gabriel Cano was born in Villanueva de Córdoba, Spain, in January 1964. He received the degree of Licenciado in physics in September 1988 from the University of Seville, Spain. He is currently in a Ph.D. program in microwaves with a scholarship from the Spanish Government.

His research interests focus on numerical methods for multiconductor planar transmission lines in MIC and MMIC.

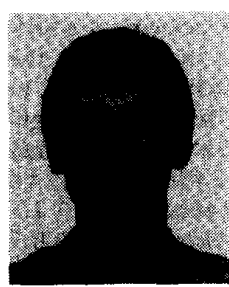

Francisco Medina (M'90) was born in Puerto Real, Cádiz, Spain, on November 9, 1960. He received the Licenciado degree in September 1983 and the Doctor degree in 1987, both in physics from the University of Seville, Spain.

$\mathrm{He}$ is currently Associate Professor of Electricity and Magnetism in the Department of Electronics and Electromagnetics, University of Seville. His research deals mainly with numerical methods for planar structures and multiconductor lines.

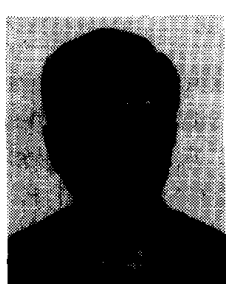

Manuel Horno (M'75) was born in Torre de Campo, Jaén, Spain. He received the degree of Licenciado in physics in June 1969, and the degree of Doctor in physics in January 1972, both from the University of Seville, Spain.

Since October 1969 he has been with the Department of Electricity and Electronics at the University of Seville, where he became an Assistant Professor in 1970, Associate Professor in 1975 and Professor in 1986. He is a member of Electromagnetism Academy of MIT, Cambridge, MA. His research interests include boundary value problems in electromagnetic theory, wave propagation through anisotropic media, and microwave integrated circuits. He is presently engaged in the analysis of planar transmission lines embedded in anisotropic materials, multiconductor transmission lines, and planar slow-wave structures. 